\title{
Doppelankauf der „Einschiffung nach Kythera“ von Watteau - zur Persönlichkeitsrechtsverletzung durch eine kunsthistorische Aussage
}

\author{
Landgericht Berlin, Urteil vom 13. November 2012 - 27 O 500/12
}

Wird auf einen in Vergessenheit geratenen Verkauf von Kunstgegenständen und somit auf einen doppelten Ankauf dieser Gegenstände (u.a. „Einschiffung nach Kythera“ von Watteau) durch den Staat aufmerksam gemacht, so ist der Erbe der Verkäufer von dieser Aussage nicht in seinem Persönlichkeitsrecht betroffen. (Leitsatz der Redaktion)

\section{Tatbestand}

- Der Kläger ist der Ururenkel des letzten deutschen Kaisers Wilhelm II. und nimmt für sich in Anspruch „als Haupterbe des Nachlasses des Hauses Hohenzollern eingesetzt" zu sein.

Die Beklagte ist Verlegerin des vom Streithelfer zu 1) verfassten Buchs „Das Berliner Schloss - Die erhaltene Innenausstattung - Gemälde, Skulpturen, dekorative Kunst", das die Geschichte der erhaltenen Bestandteile der Innenausstattung des Berliner Schlosses bis in die Gegenwart darstellt. U.a. finden dort folgende Gegenstände Erwähnung, die sich einst im Eigentum des preußischen Königshauses befanden, nämlich das Gemälde „Die Einschiffung nach Kythera" von Antoine Watteau, der Dianapokal von Hans Petzolt und der Kaiserpokal von Wenzel Jamnitzer mit den nachfolgend in Kopie wiedergegebenen Bildbeschreibungen.

Der Kläger sieht sich als öffentlicher Vertreter und Haupterbe des Hauses Hohenzollern durch die falsche Darstellung der Ankaufsgeschichte dieser drei Gegenstände in seinem Persönlichkeitsrecht verletzt. Es habe keinen Doppelverkauf gegeben. Der erste Verkauf der "Einschiffung" und der beiden Pokale vom Haus Hohenzollern an den Preußischen Staat sei nicht im Zuge der Auseinandersetzung zwischen dem ehemaligen Königshaus und dem Preußischen Staat nach der Abdankung des Preußischen Monarchen und Deutschen Kaisers im Jahre 1926/1927 erfolgt, sondern erst- und einmalig nach dem 2. Weltkrieg. Ein im Jahre 2007 aufgefundenes Dokument aus dem Jahre 1928 belege nicht den behaupteten Verkaufsvorgang, sondern stelle nur einen Entwurf des Preußischen Finanzministeriums dar, welcher nie vollzogen worden sei. Der schwere Vorwurf, das Haus Hohenzollern habe Kunstgegenstände doppelt verkauft, sei rufschädigend und genüge nicht wissenschaftlichen Ansprüchen.

\section{Der Kläger beantragt:}

Der Beklagten wird es bei Meidung eines Ordnungsgeldes in Höhe von bis zu 250.000 Euro, ersatzweise Ordnungshaft, oder Ordnungshaft bis zu sechs Monaten, untersagt, in Bezug auf den Kläger und das von ihm angetretene Erbe des Hauses Hohenzollern sinngemäß oder wörtlich zu äußern:
1. 1926 erwarb der Staat schließlich das Gemälde „Die Einschiffung" des Malers Watteau von den Hohenzollern für 1,8 Millionen Reichsmark, ein Vorgang, der über die nachfolgenden Kriegs- und Nachkriegsjahre anscheinend in Vergessenheit geriet. Denn 1983 kaufte es die Berliner Schlösserverwaltung den Hohenzollern versehentlich ein zweites Mal ab, nunmehr für 15 Millionenen D-Mark.

2. Der Dianapokal von Hans Petzolt sowie der Kaiserpokal von Wenzel Jamnitzer seien in Unkenntnis der Dokumente, die einen Ankauf durch den preußischen Staat belegen, 1963 ein zweites Mal von der öffentlichen Hand erworben worden.

Hilfweise wird beantragt, die Beklagte wie folgt zu verurteilen:

Der Beklagten wird es bei Meidung eines Ordnungsgeldes in Höhe von bis zu 250.000 Euro, ersatzweise Ordnungshaft, oder Ordnungshaft bis zu sechs Monaten, untersagt, in Bezug auf den Kläger und das von ihm angetretene Erbe des Hauses Hohenzollern sinngemäß oder wörtlich zu äußern:

1. 1926 erwarb der Staat schließlich das Gemälde "Die Einschiffung" des Malers Watteau von den Hohenzollern für 1,8 Millionen Reichsmark, ein Vorgang, der über die nachfolgenden Kriegs- und Nachkriegsjahre anscheinend in Vergessenheit geriet. Denn 1983 kaufte es die Berliner Schlösserverwaltung den Hohenzollern versehentlich ein zweites Mal ab, nunmehr für 15 Millionen D-Mark.

sowie

2. Der Dianapokal von Hans Petzolt sowie der Kaiserpokal von Wenzel Jamnitzer seien in Unkenntnis der Dokumente, die einen Ankauf durch den preußischen Staat belegen, 1963 ein zweites Mal von der öffentlichen Hand erworben worden.

ohne auf Quellen hinzuweisen, die den unter 1. und 2. geäuBerten Behauptungen widersprechen.

Die Beklagte und ihre Streithelfer beantragen,

die Klage abzuweisen. 
Ihres Erachtens entsprechen die streitgegenständlichen Behauptungen zum Verkaufsvorgang von 1926/1927 dem gegenwärtigen Stand der Wissenschaft. Sie seien durch die verfügbaren historischen Quellen belegt. Hinsichtlich des Forschungsstands im Einzelnen wird auf die Seiten 2 bis 20 des Schriftsatzes des Streithelfers zu 1) vom 25. September 2012 verwiesen. Der Kläger sei ohnehin nicht aktivlegitimiert, da er in keiner Weise von den beanstandeten Äußerungen betroffen sei. Auf postmortalen Persönlichkeitsschutz könne sich der Kläger auch nicht berufen, abgesehen davon, dass er dies bisher nicht getan habe und etwaig betroffene verstorbene Personen gar nicht konkret benenne. Grobe Entstellungen des Lebensbildes eines Verstorbenen seien nicht gegeben. Zur Ausübung postmortalen Persönlichkeitsschutzes sei der Kläger auch gar nicht berechtigt, sämtliche Schutzfristen seien zudem verstrichen.

Die beanstandeten Äußerungen seien im Übrigen in einer wissenschaftlichen Publikation in Wahrnehmung berechtigter Interessen getätigt worden.

\section{Entscheidungsgründe}

Die Klage ist unbegründet. Der Kläger hat mangels individueller Betroffenheit keinen Anspruch auf Unterlassung aus $\S \S 823$ Abs. 1, Abs. 2, analog 1004 Abs. 1 S. 2 BGB iVm Art. 1 Abs. 1, 2 Abs. 1 GG, §§ 185 ff StGB gegen die Beklagte.

Ansprüche nach § 823 BGB wegen Verletzung des Persönlichkeitsrechts stehen nur dem von der Äußerung unmittelbar Betroffenen zu. Dies folgt aus der erforderlichen Abwägung der beteiligten Grundrechte und auch daraus, dass es gilt, eine Popularklage auszuschließen.

Der Kläger selbst findet in den streitgegenständlichen Beschreibungen keinerlei Erwähnung, was nicht verwundert, weil beide geschilderten Verkaufsvorgänge vor seiner Zeit (als Chef des Hauses Hohenzollern) datieren und ihm keinerlei unmittelbare Mitwirkung an selbigen zugeschrieben wird.

Gegen rechtsverletzende Eingriffe in das Persönlichkeitsrecht kann aber nur der unmittelbar Verletzte, nicht auch derjenige vorgehen, der von den Fernwirkungen eines Eingriffs in das Persönlichkeitsrecht eines anderen nur mittelbar belastet wird, solange diese Auswirkungen nicht auch als Verletzung des eigenen Persönlichkeitsrechts zu qualifizieren sind. Insoweit gilt für das Persönlichkeitsrecht unbeschadet seiner Ausbildung als ein erst durch Güterabwägung und Interessenabwägung im Einzelfall zu ermittelnden Schutzgut nichts anderes als für die in $\S 823$ Abs. 1 BGB genannten Rechtsgüter und absoluten Rechte ( $B G H$ GRUR 1980, 813 - Familienname). In dieser Entscheidung führt der Bundesgerichtshof zum Bruder eines als Straftäter namentlich Genannten Folgendes aus, zitiert nach juris dort Rn. 12:
Freilich kann durch eine Presseberichterstattung in seiner Persönlichkeit in diesem Sinn unmittelbar betroffen nicht nur sein, wer im Mittelpunkt der Veröffentlichung steht oder auf wen sie zielt. Ein Pressebericht über einen Straftäter kann je nach Art und Inhalt der Darstellung durchaus auch andere Tatbeteiligte oder auch Angehörige des Täters in ihrem Persönlichkeitsrecht unmittelbar verletzen, wenn ihre eigenen persönlichen Verhältnisse in den Bericht einbezogen werden; sei es auch erst vermittelt durch die Person des Straftäters, etwa durch die Darstellung seiner Lebensverhältnisse und Beziehungen, die auf jenen Dritten hinweisen. Doch muß in solchen Fällen die Persönlichkeitssphäre des Dritten selbst als zum Thema des Berichts zugehörig erscheinen, damit das Erfordernis der Unmittelbarkeit noch gewahrt bleibt. Nicht genügen kann, wenn der Dritte wegen seiner engen Beziehung zu dem Straftäter durch eine Berichterstattung, die ihn selbst nicht - sei es ausdrücklich oder stillschweigend erwähnt, sich „persönlich“ betroffen fühlt. Ebensowenig reicht aus, daß Leser den beanstandeten Bericht über eine Straftat zum Anlaß nehmen, Angehörige des Täters zu belästigen oder anzufeinden, wie dies nach den Feststellungen des Berufungsgerichts im Streitfall geschehen ist. Solche Ausstrahlungen auf die Person des Dritten, in denen sich gar nicht der Inhalt der Veröffentlichung, sondern nur noch die persönliche Verbundenheit zu der in die Öffentlichkeit gerückten Person ausdrückt, bleiben als bloße Reflexwirkungen schutzlos. Anderes würde die Presse in der freien Berichterstattung ohne Sachgrund übermäßig belasten. Zwar hat sie angesichts der weitreichenden Wirkungen ihrer Veröffentlichungen größere Rücksichten auf die Betroffenen zu nehmen als der gewöhnliche Bürger in Ausübung seines Grundrechts auf Meinungsfreiheit. Schutzwürdig ist jedoch auch ihr Anliegen, den Kreis der durch ihre Veröffentlichungen berührten Persönlichkeitsinteressen überschaubar zu halten.

Vorliegend war der Kläger offensichtlich und für den Leser erkennbar nicht familienintern in den Verkauf der Kunstgegenstände verstrickt. Die Berichte erörtern weder ausdrücklich noch „zwischen den Zeilen“ die Person des Klägers.

Der Kläger ist auch nicht etwa deshalb betroffen, weil er sich selbst zum bekannten Haus Hohenzollern zählt. Die Bildnebenschriften zielen selbstverständlich auf die damaligen Akteure im Hause Hohenzollern ab. Allein in seiner Eigenschaft als Nachfahre seiner bekannten Vorfahren wird er in Person nicht jedesmal bei der Erwähnung des Hauses Hohenzollern im Zusammenhang mit historischen Betrachtungen zum unmittelbar Betroffenen. Selbst wenn die vorliegenden Beiträge geeignet sein sollten, beim Leser Vorurteile zu den Nachfahren des Hauses Hohenzollern zu wecken, was die Kammer nicht zu erkennen vermag, machte ihn dies nicht zum unmittelbar Betroffenen.

Dass der Name Hohenzollern nicht ein beliebiger ist, ist unschädlich. Dass Inhalt und Reichweite des Persönlichkeitsschutzes nicht von der Prägekraft eines Familiennamens abhängig gemacht werden kann, liegt auf der Hand. Insoweit verwirklicht sich nur das Allgemeine Lebensrisiko des Trägers eines ungewöhnlichen Familiennamens (vgl. BGH aaO). 
Der Kläger zählt sich offenbar selbst nicht zum Kreis der unmittelbar Verletzten, sondern vielmehr seine "Familie" bzw. das „Haus Hohenzollern“.

Der Streitfrage, ob der Familie ein eigenständiges Persönlichkeitsrecht zustehen kann (was nach der Rechtsprechung des Bundesgerichtshofs ohnehin Bedenken unterliegt - BGH GRUR 1974,794 - Todesgift; GRUR 1974, 797 - Fiete Schulze; NJW 1969, 1110 - Detektei - und vom Kammergericht im Beschluss v. 27.4.1992 - 9 W 2297/92 - verneint wird), muss vorliegend nicht nachgegangen werden; denn sie ist jedenfalls in einem so umfassenden Sinn, dass nunmehr der Ururenkel des letzten deutschen Kaisers hieraus Ansprüche geltend machen könnte, nicht Gegenstand der Veröffentlichung gewesen. Mit der damaligen Vermögensauseinandersetzung mit dem Preußischen Staat nach der Abdankung des Preußischen Monarchen haben die Nachfahren des Hauses Hohenzollern, darunter der Kläger, längst nichts mehr zu tun. Auf eine etwaige Verletzung der Familienehre kann er sich heute nicht mehr berufen, sodass dahinstehen kann, ob er überhaupt Träger einer solchen wäre.

Der Kläger ist auch als derzeitiger Repräsentant des Hauses Hohenzollern nicht betroffen, weil Vorwürfe gegen verstorbene Mitglieder des Hauses erhoben werden.

Dies wäre allenfalls dann in Erwägung zu ziehen, wenn es sich nicht wie hier lediglich um historische Betrachtungen handelte, sondern der jetzige Chef des Hauses Hohenzollern durch solche Kritik aktuell beeinträchtigt würde. Letzteres ist offensichtlich nicht der Fall. Mitnichten wird hier dem Nachfahren des Hauses mit den beanstandeten Bildbeschreibungen Geldgier oder die Bereicherung auf Kosten des Steuerzahlers nachgesagt, sondern lediglich auf einen scheinbar historisch in Vergessenheit geratenen Verkauf von Kunstgegenständen im Rahmen der Vermögensauseinandersetzung mit dem Preußischen Staat nach Abdankung des Preußischen Monarchen und Deutschen Kaisers aufmerksam gemacht. Eine aktuelle Beeinträchtigung des Klägers geht damit keineswegs einher.

Die Betroffenheit des Klägers folgt auch nicht aus der eingereichten Presseberichterstattung. Im Artikel aus der Märkischen Allgemeinen findet er gar keine Erwähnung. „Bild.de“ benennt ihn lediglich als Kläger im hiesigen Rechtsstreit.

Der Kläger kann sich hier auch nicht auf postmortalen Persönlichkeitsschutz berufen.

Der rechtliche Schutz der Persönlichkeit gemäß Art. 1 Abs. 1 GG endet nicht mit dem Tode. Vielmehr besteht der allgemeine Wert- und Achtungsanspruch fort, sodass das fortwirkende Lebensbild eines Verstorbenen weiterhin gegen schwerwiegende Entstellungen geschützt wird. Dieser Schutzanspruch kann von den nächsten Angehörigen geltend gemacht werden (BGH NJW 1968, 1773, 1774 f. - Mephisto; BVerfG NJW 1771, 1645, 1646 f. Mephisto; BGH WRP 1984, 681, 682 - Frischzellenkosmetik).
Vorliegend ist das Lebensbild keines verstorbenen Hohenzollern schwerwiegend entstellt. Wer überhaupt aus dem Hause Hohenzollern den - allenfalls fragwürdigen und unter Ehrschutzaspekten zu prüfenden - „zweiten Verkauf“ der Kunstgegenstände zu verantworten hatte, bleibt gänzlich offen, ist den beanstandeten Äußerungen mit keinem Wort zu entnehmen und vermag offenbar nicht einmal der mit der "Familiengeschichte“ vertraute Kläger anzugeben. Sollte hierfür überhaupt eine verstorbene Person verantwortlich zeichnen, deren postmortale Ansprüche der Kläger geltend zu machen befugt ist, fehlte es jedenfalls an einer Entstellung des Lebensbildes oder Verletzung der Menschenwürde dieser wohl kaum erkennbaren Person, weil jener mit dem lapidaren Hinweis auf den versehentlichen Doppelkauf durch den Staat keinerlei Vorsatz oder gar Täuschungsabsicht unterstellt wird. (Entscheidung von der Redaktion bearbeitet) 


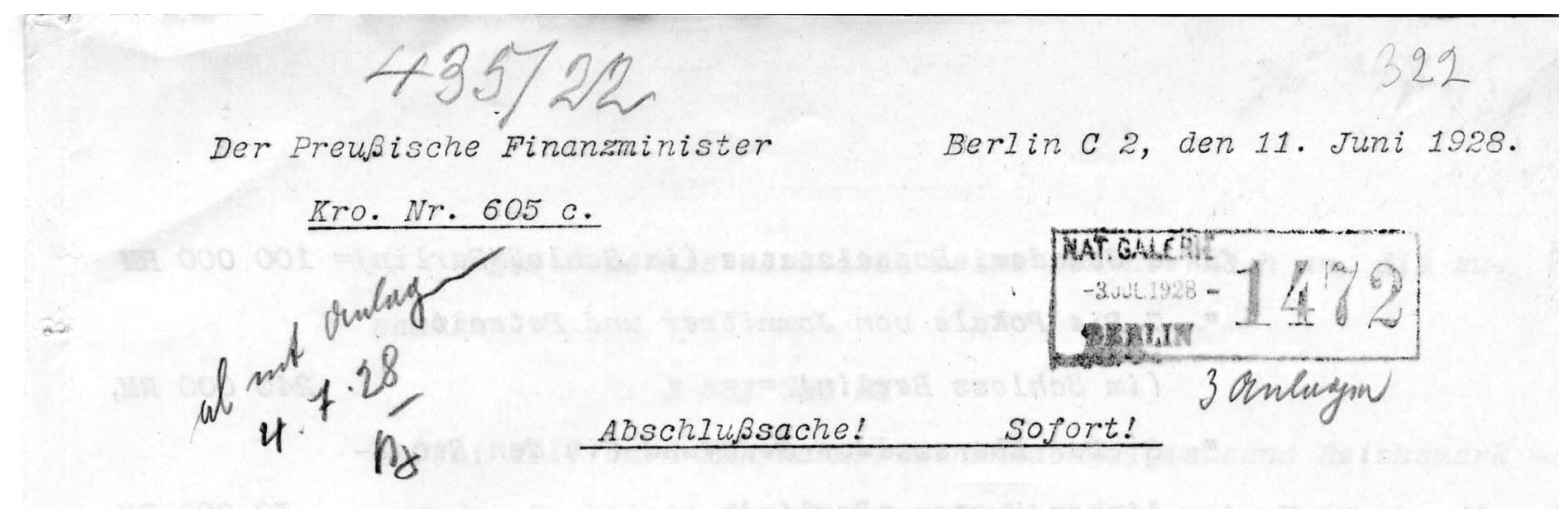

Nach Massgabe des $\$ 2$ ziffer IV Absatz 3 und der Anlage $B$ Abschnitt III des Vertrages über die Vermögensauseinandersetaung zwischen dem Preussischen Staate und den Mitgliedern des vormals regierenden Preussischen Königshauses von 12. 10. 1925, beide in der Fassung des Abänderungsvertrages zu diesem Vêtrage vom 6. 10. 1926 - Ges. S. 1926 Seiten 279 und 287 - ist awischen dem staate und dem vormals regierenden Preussischen Königshause zunächst mïndlich vereinbart und dann unter dem 18. 11. 1926 durch die hier beigefügten Anlagen 1 und 2 schriftlich bestätigt worden, daß das Eigentum an den in der obengenannten Vertrags-Anlage $B$ in Abschnitt III unter Ziffern 1 bis 10 und 14 bis 19 aufgeführten Kunstwerke auf den Preussischen Staat übergegangen ist, wogegen vom Preubischen Staat als Entgelt fiir diese Kunstwerke die von dem gemeinsam emannten Sachverständigen Geh. Regierungsrat Proj. Dr. Friedländer in Berlin bestimmten Kaufpreise und swar:

jür 1)Watteau, Einschiffung aur Liebesinsel (im SchIOßBerlin) $=$ $1800000 \mathrm{PM}$

" 2) Fring Wandteppiche im Stile Le Bruns (im SchIoss Charlottenburg) $=150000$ RM,

" 3) Bouchardon, Büste Karis XII (im Schloß
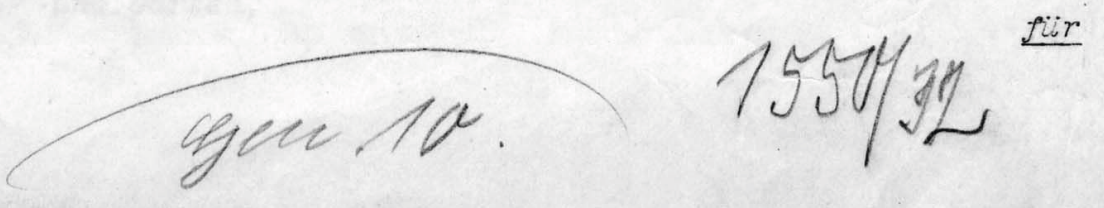
fïr 6 Schadow, Doppelstatue (im SchIo, Berlin) $=100000$ RII

" 7 Die Pokale von Jamnitzer und Petzolt

(im Schloss Berlin) $=$ 240000 RII,

" 8 Der Eberswalder Goldfund (in den Staat-

Iichen Uuseen, Berlin)= 50000 RLI

" 9 Die Ausgrabungen der Herzogin Paul von Hecklenburg in Kärnten und Krain (in den Staatlichen Iuseen Berlin )= 25000 RHL

" Pater, Badende Mädchen und Tanz im Freien (Seide1: Französische Kunstwerke Nr. 87/88/ (im Neuen Palais) $=$ 360000 RMI

" 14 Tonbüste der Komtesse de Sabran und Gegenstiuck $=$ 250000 RM

" 15 Räntgenuhr (im Schloss Berlin) = 40000 RIII

" 16 Zehn Sèvresstatuetten (im Schloß Berlin) 50000 RM

" 17 Hamburger Schrank (im Schloss Berlin) $=6000$ RM

" 18 Lieberkïhnscher Humpen (im Schloß Berlin) 30000 RII

und " 19 Silberne Tragfigur (im Schloß Berlin) = 20000 RIII zusammen: $\quad 3581000$ RLL. an das vormals regierende Preussische Königshaus, mit 2000000 RM bis spätestens 30. 11. 1926 und mit den restlichen 1581000 RM bis spätestens 2. 4.1927 zu zahlen waren. Beide Beträge sind vorbehaltlich der später erst zu regeInden endgüItigen Verrechnung aus dem Konto $I$ bei der Preussischen Staatsbant (Seehandlung) gezahlt worden und zwar die 2000000 Rill nach den Angaben auj Anlage 1 und der als Anlage 3 beigefiigten quittung am 19. 11. 1926, die 1581000 RM nach den Angaben der Anlage 4 und der als Anlage 5 beigefügten quittung am 19. 3. 1927. Die endgïltige Verrechnung soll nunmehr zur Rechnung fïr 1927 erfolgen. 


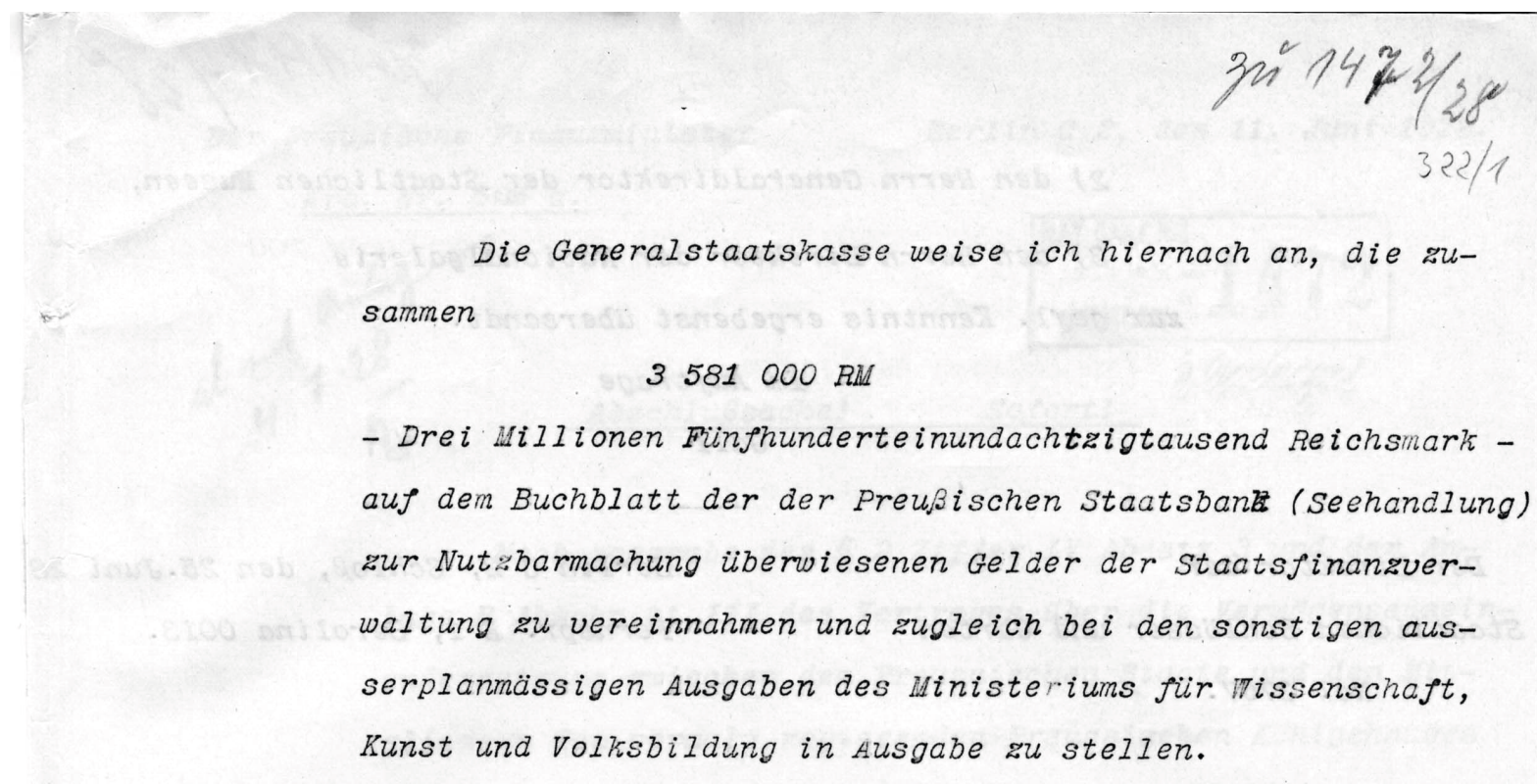

In vertretung

An die Generalstaatskasse

Vorstehende Abschrift gestatte ich mir zur gefälligen Kenntnisnahme mit dem Hinzufügen ergebenst zu übersenden, daß ich die Anweisung ohne dortige Mitzeichnung habe erteilen müssen, weil anders die Hineinbringung der jetzt erst wieder zur Sprache gekommenen Zahlung der 3581000 RM in den Abschlus für 1927 nicht mehr möglich gewesen wäre.

Festgestellt

gez. Winter

Antsrat

\section{In Vertretung}

gez. $S c h l$ e us e ner

An den Herrn Preußischen Minister für Wissenschaft, Kunst und Volksbildung

Der Preusische Minister Berlin WI 8, den 19. Juni 1928. für Wissenschaft, Kunst und Volksbildung.

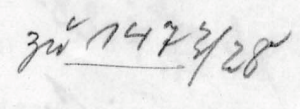

U. IV Nr. $25259 / 28$.

Urschriftlich mit 1 Anlage $u$. R.

an 1. den Herrn Direktor der Staatlichen Schlösser und Gärten,

2) 


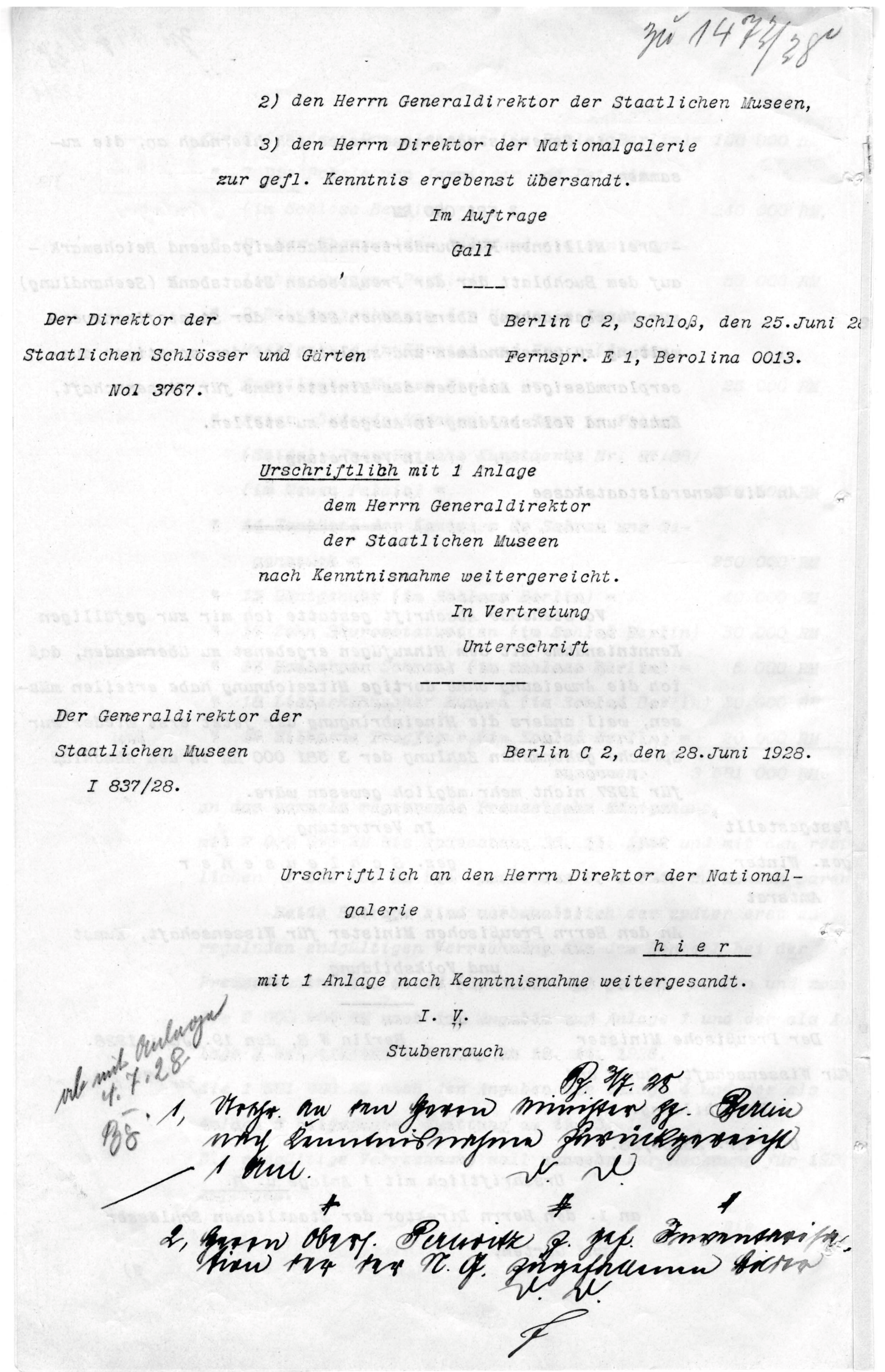

